\title{
Biologically inspired signal processing: analyses, algorithms and applications
}

\author{
Elias Aboutanios ${ }^{*}$, Steve McLaughlin ${ }^{2}$, Yannis Kopsinis ${ }^{3}$ and Raviraj Adve ${ }^{4}$
}

"How fleeting are the wishes and efforts of man! How short is his time! And consequently how poor will his products be, compared with those accumulated by nature during whole geological periods. Can we wonder, then, that nature's productions should be far 'truer' in character than man's productions; that they should be infinitely better adapted to the most complex conditions of life, and should plainly bear the stamp of far higher workmanship?" - Charles Darwin, The Origin of Species.

Many of the problems that are encountered in Engineering have been solved in nature. In their quest for survival species have developed ingenious solutions to these problems over millions of years. As Darwin noted, these solutions that natural evolutionary processes have and are still in the process of perfecting, are in most cases far superior to what we can achieve with our current engineering knowledge and methods. This should come as no surprise when one considers the power of natural selection and the vast time span and enormous scale of possibilities that it operates over.

The superiority and inspirational qualities of naturally evolved solutions certainly hold true in the field of signal processing. In their quest for survival within their natural environment, living organisms must sense this environment, which they carry out using visual, auditory, tactile, olfactory and gustatory sensors. They must then process the acquired signals and respond to the cues they extract from them. Often these signals originate from the environment, but many organisms have also evolved the ability to produce stimuli or transmit signals that permit them to probe their surroundings. Thus living creatures have not only acquired advanced signal processing systems but have also achieved adapted waveform designs that, combined with the sensors and signal processing systems, lead to astounding levels of performance.

\footnotetext{
* Correspondence: elias@ieee.org

'The school of Electrical Engineering and Telecommunications, the University of New south Wales, Sydney, Australia

Full list of author information is available at the end of the article
}

Consider, for instance, the problems of navigation and object detection, localisation and tracking. Radar and Sonar provide solutions to these problems and have become essential to the functioning of our society. While these relatively new technologies may arouse the wonder of people at the capabilities of humans, they nonetheless pale in comparison to the achievements of bats in these domains. This is even more amazing when one remembers that bats have, for millions of years, been employing ultrasonic waves to navigate, forage, locate and hunt their prey with great efficacy. That they do this with great success is a matter that is crucial for their survival.

As we improve our knowledge of our natural environment and continually discover the wonders it has evolved, it is important to draw inspiration from it. Understanding how nature implements what it has perfected over such a long time, what solutions it has selected and those it has discarded, will surely lead to significant advances in Signal Processing Research. These observations not only apply to the highly advanced signal processors, but also to the sensors as well as the waveforms that are exquisitely adapted to their purpose and in many cases amount to optimally integrated signal processing systems. The papers that are published in this special issue describe research results in sensors, signals and signal processing algorithms that are inspired by naturally evolved analogues.

Many animals depend for their survival on detecting and locating objects, whether these objects are obstacles for navigation or prey that is hunted. Dolphins are known to have evolved highly advanced detectors that are capable of discriminating objects both below the surface and on the seabed. Bio-inspiration is used in paper (484619) to improve the performance of man-made sonar in the detection of marine cables by adopting dolphin-like wideband sonar pulses. In addition to radar and sonar, biologically inspired waveform designs can play an important role in many areas such as Ultrasound Contrast Imaging (UCI). However, these waveforms often 
consist of short duration pulses that require localised processing. The Discrete Fourier Transform (DFT) is a global transform, and the performance of DFT-based algorithms suffers when they are applied to time-varying and non-stationary signals such as those encountered in biological systems. These issues are examined in the next three papers. The authors of (146175) present a Bayesian framework that is capable of estimating the signal parameters and which significantly outperforms DFT-based techniques. In (532349) the authors use relevance analysis of random bio-signals to identify pathologies; their sample application is the diagnosis of obstructive sleep apnea. In (284791) the application is automatic speech recognition based on phoneme classification. The authors develop an evolution-based spline functions approach as the fitness measure leading to an optimized filterbank for phoneme classification.

The efficient waveform designs found in living organisms are matched by equally well-adapted biological sensors. The work reported in article (806406) is concerned with autonomous underwater vehicles where an advanced array of artificial hair-cell flow sensors is developed that, with the aid of a specialized signal-processing unit, is imitating the sophisticated near-field sensing capability of fish.

Vision is arguably the most important and heavily relied upon sensory system in living organisms. Furthermore, its amazing capabilities are leaps and bounds above anything currently achievable by technological means. Therefore, it is hardly surprising that it has and continues to be an important source of bio-inspiration. In article (781561) the authors draw on the human visual system for inspiration to enhance the measurement of object motion. They demonstrate that the developed framework is capable of dealing with real image sequences. Articles (101428), (841078) and (145232) apply knowledge and models of the visual system in robotics. In (101428) the author presents a new object categorization method that uses a biologically motivated codebook selection approach to break the image into its constituent objects and background. In paper (841078) the authors study social robots that are intended to interact with humans. They develop an object-based attention mechanism that is able to focus the resources of the robot to the object that is relevant to the current task. Article (145232) applies bio-inspiration from the visual system to advance the area of autonomous underwater vehicles and robots respectively. In particular, a visual landmark detection system is developed by modelling object shapes as cylinders inspired by Marr's visual theory.

The inspiration that can be drawn from living organisms is by no means limited to their organs or subsystems. External behaviour, whether individual or group based, has also been evolving to serve the species' survival. Therefore, in article (341836) a novel clusteringbased non-coherent ultra wide band (UWB) detector is proposed which is relying on a biologically inspired Particle Swarm Optimization approach.

Finally, in paper (973806), the authors look to the evolutionary principles themselves for inspiration. Thus they formulate an efficient FPGA-based approach to optimize a wavelet transform for improved image compression.

It is clear that like nature biologically inspired signal processing algorithms will continue to evolve and we hope that this issue provides a useful snapshot of research in our community and will inspire researchers to continue to explore this space.

\section{Author details}

${ }^{1}$ The school of Electrical Engineering and Telecommunications, the University of New south Wales, Sydney, Australia ${ }^{2}$ The Institute for Digital Communications, the University of Edinburgh, Edinburgh, The UK ${ }^{3}$ The Department of Informatics and Telecommunications, The University of Athens, Athens, Greece ${ }^{4}$ The Edward S. Rogers Sr. Department of Electrical and Computer Engineering, The University of Toronto, Toronto, Canada

Received: 14 April 2011 Accepted: 14 February 2012 Published: 14 February 2012

doi:10.1186/1687-6180-2012-28

Cite this article as: Aboutanios et al:: Biologically inspired signal processing: analyses, algorithms and applications. EURASIP Journal on Advances in Signal Processing 2012 2012:28.

\section{Submit your manuscript to a SpringerOpen ${ }^{\circ}$ journal and benefit from:}

- Convenient online submission

- Rigorous peer review

- Immediate publication on acceptance

- Open access: articles freely available online

- High visibility within the field

- Retaining the copyright to your article

Submit your next manuscript at $>$ springeropen.com 